\title{
Sub-decibel off-chip fiber couplers based on $L$-shaped waveguides and subwavelength grating metamaterials
}

\author{
D. Benedikovic ${ }^{1, *}$, C. Alonso-Ramos ${ }^{1}$, S. Guerber ${ }^{1,2}$, D. Pérez-Galacho ${ }^{1,3}$, X. Le Roux ${ }^{1}$, V. Vakarin ${ }^{1}$, G. Marcaud ${ }^{1}$, P. \\ Cheben $^{4}$, D. Marris-Morini' ${ }^{1}$, E. Cassan ${ }^{1}$, C. Baudot ${ }^{2}$, F. Boeuf ${ }^{2}$, and L. Vivien ${ }^{1}$ \\ ${ }^{1}$ Centre de Nanosciences et de Nanotechnologies, CNRS, Univ. Paris-Sud, Université Paris-Saclay, C2N - Palaiseau, 91120 \\ Palaiseau, France \\ ${ }^{2}$ STMicroelectronics, Silicon Technology Development, 38926 Crolles, France
}

${ }^{3}$ Photonics Research Labs, iTEAM Research Institute, Universitat Politecnic de Valencia, Spain

${ }^{4}$ National Research Council Canada, Ottawa, ON K1A 0R6, Canada

*daniel.benedikovic@c2n.upsaclay.fr

\begin{abstract}
Uniform grating couplers based on versatile $L$ shaped waveguides are experimentally demonstrated, with coupling loss of $-2.7 \mathrm{~dB}$ and back-reflections of $-20 \mathrm{~dB}$. Apodized couplers with subwavelength-grating metamaterials predict improved fiber-chip coupling down to $-0.46 \mathrm{~dB}$ within device layouts compatible with lithographic technologies available in nanophotonic foundries.
\end{abstract}

Keywords - silicon nanophotonics, sub-wavelength grating metamaterials, silicon-on-insulator, grating couplers, deepultraviolet technology, mass-scale production

\section{INTRODUCTION}

The success of silicon nanophotonic technologies hinges upon availability of low-loss input / output optical interfaces. Off-chip fiber couplers are particularly interesting as they enable wafer-scale device testing and provide improved alignment margins in fiber attachments [1-3].

Conventional grating couplers call upon advanced design strategies with adjusted vertical layer stack. This includes Bragg / metal mirrors underneath [4,5], subsidiary overlays [6], or multi-level chip architectures [7]. However, such approaches fall apart, since they are either not realizable in mature CMOS foundries, involve custom substrates and procedures, lack straightforward integration into the existing or future networks, or arguably have scaling limitations.

In stark contrast, dual-etch fiber-chip grating couplers with interleaved [8] or $L$-shaped waveguide geometries [9-11] have emerged as a unique solution for coupling of light between on-chip submicron waveguides and standard optical fibers. Such couplers advantageously exploit blazing effect in a scalable fashion by using only two lithography and etching procedures. The superior radiation performance is fully controlled via asymmetric scatterers that yield constructive (destructive) interference in the up (down) direction. Blazed dual-etch grating couplers are facile to design and fabricate using existing processes. They are easily portable to other silicon [8-10] or even non-silicon waveguide platforms [11].

Here, we report on recent results in development of efficient $L$-shaped surface grating couplers, owning device layouts readily compatible with lithographic technologies, such as deep-ultraviolet (deep-UV) steppers, widely available in silicon nanophotonic foundries.

\section{UNIFORM GRTAING COUPLERS}

Figure 1a shows a general schematic of the uniform fiberchip grating coupler with an $L$-shaped waveguide radiation profile. Devices were deigned on SOI substrates, with 300nm Si layer and 720-nm BOX. They operate with TE-like waveguide mode at wavelength of $1.55 \mu \mathrm{m}$. Coupler geometry features full- and partial-etch trenches and unetched $\mathrm{Si}$ slabs to achieve an outstanding radiation performance that maximizes optical power coupled into fiber, whereas scaling down the parasitic radiation into Si substrate. Rigorous 3D FDTD calculations predict remarkably high directionality, exceeding $98 \%$ for a wide range of grating geometries across a $100 \mathrm{~nm}$ wavelength range [9]. Grating couplers also include a short metamaterial transition stage to reduce the reflectivity at the waveguide-to-grating juncture. This was implemented as periodic array of $\mathrm{Si}$ pillars structured at subwavelength grating (SWG) scale, here oriented perpendicular to direction of light propagation. SWG transition yields reflectivity reduction from $16 \%$ to $2 \%$. Fiber-chip coupling loss of $-2.2 \mathrm{~dB}$ were predicted for both designs without and with SWG metamaterial transition.

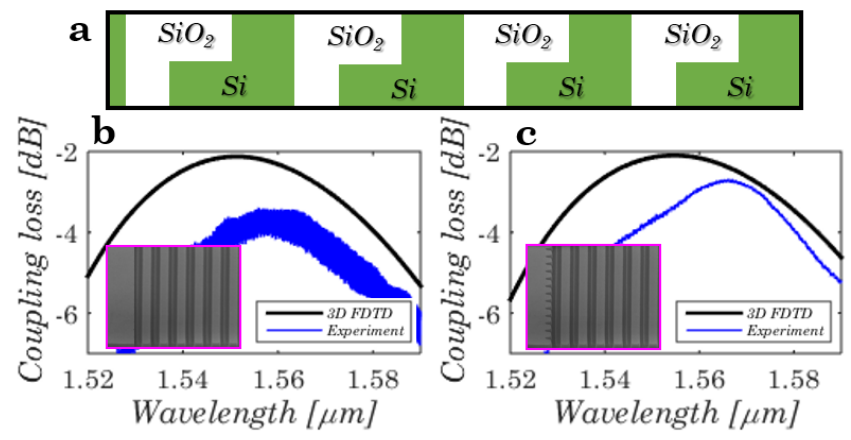

Fig. 1 (a) Schematic of a uniform $L$-shaped fiber-chip grating coupler. Calculated and measured coupling loss as a function of a wavelength for $L$ shaped grating couplers: (b) without and (c) with a SWG transition stage. Insets: Scanning electron microscopy images of fabricated couplers.

Grating couplers were fabricated in STMicroelectronics photonic foundry facilities using $300 \mathrm{~mm}$ SOI's and CMOS production line [9]. Figures $1 \mathrm{~b}$ and 1c compare fiber-chip 
coupling loss of designed and fabricated $L$-shaped couplers as a function of the operating wavelength. Insets show scanning electron microscopy images of fabricated devices. Peak coupling loss of $-3.4 \mathrm{~dB}$ at a wavelength of $1560 \mathrm{~nm}$ was measured for coupler design without the transition stage. Here, spectral response exhibits a distinctive Fabry-Perot ripples with amplitude of around $0.85 \mathrm{~dB}$. This yields estimated reflectivity of $\sim 8 \%$. In opposite, grating couplers with SWG transition stage reached peak loss of $-2.7 \mathrm{~dB}$ at $1565 \mathrm{~nm}$, with some residual, yet comparatively lower fringes in the spectral response. The estimated grating reflectivity is $\sim 1 \%$, i.e. $-20 \mathrm{~dB}$. This corresponds to the 8 -fold reduction in measured grating coupler back-reflections.

\section{SUB-DECIBEL GRATING COUPLERS}

Figure 2a shows a vertical schematic of the proposed $L$ shaped grating coupler apodized by SWG metamaterials. By implementing the SWG structures within the etched grating trenches, the coupling strength of the grating can be easily controlled, yet the superior radiation performance remains almost unaffected, with a directionality larger than $88 \%$. Figure $2 \mathrm{~b}$ shows $2 \mathrm{D}$ mapping of the estimated coupling loss as a function of number of apodized periods (ranging from 1 to 5) and the SWG critical dimensions (set between $0 \mathrm{~nm}$ and $200 \mathrm{~nm}$ ), as calculated at nominal wavelength of $1.55 \mu \mathrm{m}$. The mode field diameter of standard optical fiber is $10.4 \mu \mathrm{m}$. According to the numerical optimization, exceptionally low fiber-chip coupling is readily obtained for a wide range of studied grating coupler arrangements. Indeed, of particular practical interest are the critical dimensions constraints typically allowed in both academic- and industry-oriented silicon nanophotonic developments. Here, 50-nm resolution limit for exploratory electron beam (e-beam) lithography and rather conservative 100-nm limit for large-volume deep-UV optical technology, were considered.

To illustrate the versatility of the proposed approach, an $L$ shaped grating coupler with only a 1 apodized period already reaches coupling loss of $1 \mathrm{~dB}$ for a SWG minimum feature size in excess of $160 \mathrm{~nm}$. In addition, an $L$-shaped grating coupler with 5 apodized periods (see Fig. 2c) provides a peak fiber-chip coupling loss of $-0.46 \mathrm{~dB}$ at a SWG critical dimension of $80 \mathrm{~nm}$. Herein, surprisingly low coupling loss penalty of only $0.35 \mathrm{~dB}$ is found for an ultra-wide range of critical dimensions for synthesized SWG metamaterials, spanning from $50 \mathrm{~nm}$ up to $200 \mathrm{~nm}$. This grating coupler design suggests an unprecedented robustness to deliver a subdecibel fiber-chip coupling reliance in respect to the given minimum feature size restrictions. For the same apodized coupler design, the corresponding evolution of grating reflectivity as a function of the SWG critical dimensions is shown in Fig. 2(d). The grating coupler reflectivity down to $20 \mathrm{~dB}$ can be obtained for practical deep-UV-compatible feature sizes.

The superior design flexibility, as jointly provided by the $L$ shaped waveguide profile and index-engineered SWG metamaterials, paves the way for robust coupling performance. This versatility also provides a suite of design freedoms to choose from towards desired scenario. Furtheremore, the lithographic and etching complexity to define coupler layout is comparatively less challenging to be proceed within established foundries, as compared to earlier grating coupler implementations [4-7].

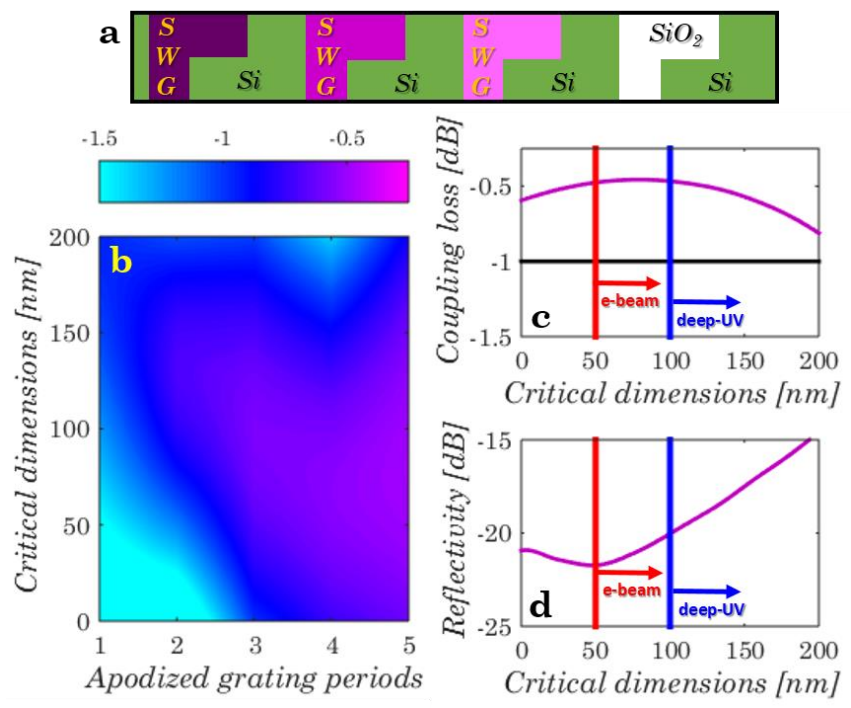

Fig. 2 (a) Schematic of apodized $L$-shaped fiber-chip grating coupler engineered by SWG metamaterials. (b) 2D mapping of the coupling loss as a function of number of apodized grating coupler periods and SWG critical dimensions. (c) Coupling loss and (d) reflectivity as a function of the SWG critical dimensions for $L$-shaped coupler design with 5 apodized periods.

\section{CONCLUSIONS}

We demonstrated versatile $L$-shaped grating couplers with synthesized SWG metamaterials. Experimentally, peak coupling loss of $-2.7 \mathrm{~dB}$ and reduced back-reflections of -20 $\mathrm{dB}$ were achieved. We also proposed an apodized fiber-chip couplers with robust sub-decibel performances and device layouts compatible with 100-nm lithographic technologies used in commercial nanophotonic foundry settings.

\section{ACKNOWLEDGMENTS}

This work was supported by the European Research Council (ERC) under the European Union's Horizon 2020 research and innovation program (ERC POPSTAR - grant agreement No 647342).

\section{REFERENCES}

[1] P. Cheben, et al., Nature 560, 565-572, (2018).

[2] R. Halir, et al., Proc. IEEE 106, 2144-2157 (2010).

[3] R. Marchetti, et al., Photon. Res. 7, 201-239 (2019).

[4] A. Mekis, et al., J. Sel. Top. Quantum Electron. 17, 597608 (2011).

[5] D. Benedikovic, et al., Opt. Express 23, 22628-22635 (2015).

[6] D. Vermeulen, et al., Opt. Express 18, 18278-18283 (2010).

[7] M. T. Wade, et al., Proc. IEEE OI 2015, paper TuB4.

[8] D. Benedikovic, et al., Opt. Lett. 40, 4190-4193 (2015).

[9] D. Benedikovic, et al., Opt. Lett. 42, 3439-3443 (2017).

[10] T. Watanabe, et al., J. Lightw. Technol. 35, 4663-4669 (2017).

[11] Y. Chen, et al., Opt. Lett. 42, 3566-3569 (2017). 